\title{
Understanding Jet Structure and Constituents: Track Jets and Jet Shapes at ATLAS
}

\author{
Seth Zenz for the ATLAS Collaboration \\ UC Berkeley and LBNL, Berkeley, CA 94720, USA \\ DOI: http://dx.doi.org/10.5689/UA-PROC-2010-09/20
}

\begin{abstract}
The ATLAS Detector at the Large Hadron Collider has begun to take proton-proton collision data, and an understanding of hadronic jets and their substructure is an important part of its early physics program. The agreement between detector simulation and uncorrected data for jet constituent multiplicities and jet shapes is illustrated. A preliminary measurement of the cross section and fragmentation of jets defined using charged particles with transverse momentum greater than $500 \mathrm{MeV}$ is presented.
\end{abstract}

\section{Introduction}

The measurement of jets is an important part of the physics program at the Large Hadron Collider (LHC). In addition to the measurement of jet cross-sections and comparison to QCD preductions, accurate jet measurements are important for high-mass particles decaying to quarks or gluons. As LHC luminosity increases and events of interest have multiple "pileup" collisions, relatively low-energy jets from these extra collisions will impact virtually every physics measurement. Jets and their structure must therefore be understood. In this work, calorimeter cluster and track multiplicity and jet shapes in data are compared with simulation. A measurement is presented of jets defined using charged particles measured by the ATLAS Inner Detector, including both the cross section and fragmentation.

\section{The ATLAS Detector}

The ATLAS Detector [1] surrounds the Large Hadron Collider proton-proton collision point with layers of tracking detectors, calorimeters, and muon chambers. The tracking and calorimeter systems, along with the minimum bias and jet triggers, are of particular interest for jet reconstruction.

High granularity liquid-argon (LAr) electromagnetic sampling calorimeters, with excellent energy and position resolution performance, cover the pseudo-rapidity range $|\eta|<3.2$. The hadronic calorimetry in the range $|\eta|<1.7$ is provided by a scintillator-tile calorimeter. In the end-caps $(|\eta|>1.5)$, LAr hadronic calorimeters match the outer $|\eta|$ limits of the end-cap electromagnetic calorimeters. The LAr forward calorimeters provide both electromagnetic and hadronic energy measurements, and extend the coverage to $|\eta|<4.9$.

The ATLAS Inner Detector provides precise track reconstruction over pseudorapidity $|\eta|<$ 2.5. It consists of three layers of pixel detectors close to the beam-pipe, four layers of silicon 
microstrip detectors (SCT) providing eight hits per track at intermediate radii, and a transition radiation tracker (TRT) at the outer radii, providing about 35 hits per track.

Except where noted, data presented here was triggered during LHC stable beams using the Minimum-Bias-Trigger-Scintillators (MBTS). These cover the pseudorapidity range, $2.09<$ $|\eta|<3.84$, and are located in front of the end-cap electromagnetic calorimeters; at least one MBTS hit is required. This trigger is nearly $100 \%$ efficient for all jet definitions.

The Level-1 calorimeter trigger is also used, with a threshold of $5 \mathrm{GeV}$. This sums the raw energy over electromagnetic and hadronic calorimeter towers in elements with a granularity of $\Delta \phi \times \Delta \eta=0.2 \times 0.2$ for $|\eta|<3.2$, then applies a sliding window algorithm to identify the regions with the greatest transverse energy deposited.

\section{Track Selection}

Tracks measured in the Inner Detector may be associated with jets measured in the calorimeter or used directly to define track jets (section 4). Tracks are reconstructed using the silicon-seeded tracking algorithm [2] and extrapolated to the TRT. They are required to have:

- Transverse momentum $p_{\mathrm{T}}>500 \mathrm{MeV}$.

- Transverse impact parameter with respect to the primary vertex $\left|d_{0}\right|<1.5 \mathrm{~mm}$.

- Longitudinal impact parameter $z_{0}$ satisfying $\left|z_{0} \sin \theta\right|<1.5 \mathrm{~mm}$.

- At least $6 \mathrm{SCT}$ hits

For tracks matched to calorimeter jets, a hit in the pixel detector is also required. For tracks used in reconstructing track jets, a hit in the innermost pixel layer is required, along with a hit in either the outer most pixel layer or the innermost SCT layer.

\section{Jet Reconstruction}

Jet properties and substructure may be investigated by applying a jet algorithm to either (a) energy clusters in the calorimeter, or (b) tracks measured in the Inner Detector. Jets are constructed by applying the anti- $k_{t}$ algorithm [3] with parameter $\mathrm{R}$ of 0.4 and 0.6 to the relevant constituents.

The input objects for calorimeter-based jets are topological energy clusters in the calorimeter [4]. These clusters are seeded by calorimeter cells with energy $\left|E_{\text {cell }}\right|>4 \sigma$ above the noise, where $\sigma$ is the RMS of the noise. All directly neighbouring cells are added, then neighbours of neighbours are iteratively added for all cells with signals above a secondary threshold $\left|E_{\text {cell }}\right|>2 \sigma$. Finally the energy in all further immediate neighbours is added. Clusters are merged based on the position of local minima and maxima. The cell energies are summed to give the cluster energy, and the clusters are treated as massless. The baseline calibration for these clusters corrects their energy to the electromagnetic (EM) scale. Further corrections for the overall jet energy scale, accounting for calorimeter non-compensation, energy losses in inactive regions of the detector, and energy losses due to charged particles curving in the solenoidal field, are described in [5]. 


\section{Jet Shapes and Constituents}

In order to evaluate the modeling of jets and their interactions with the detector, reconstructed data are compared to simulation. Events are produced using the ATLAS MC09 [6] tune of Pythia 6 [7] and passed through a GEANT [8] model of the ATLAS detector.

Figure 1 shows the number of constituents associated with jets with $p_{\mathrm{T}}$ between 20 and $100 \mathrm{GeV}$, for events triggered with one MBTS hit. The data has more clusters per jet, and more associated tracks per jet, than the simulation; however, the discrepancy is much reduced if associated tracks with $p_{\mathrm{T}}>1.0 \mathrm{GeV}$ are compared. More information on this comparison can be found in reference [5].

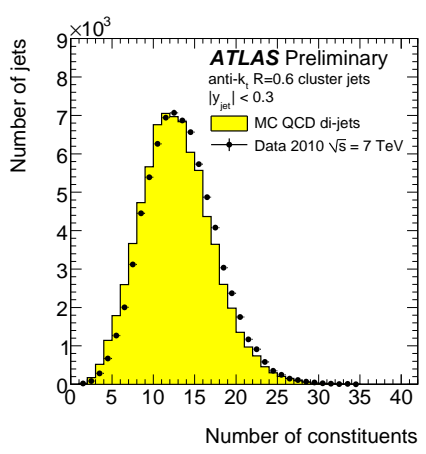

(a) Clusters

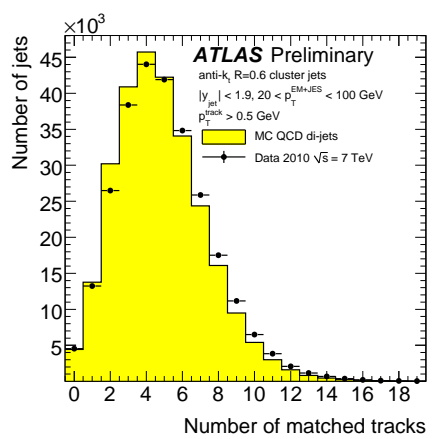

(b) Tracks, $p_{\mathrm{T}}>0.5 \mathrm{GeV}$

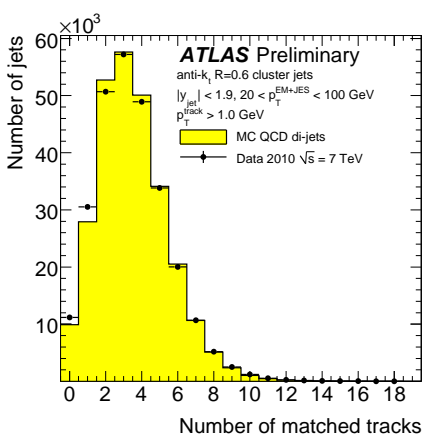

(c) Tracks, $p_{\mathrm{T}}>1.0 \mathrm{GeV}$

Figure 1: The number of constituents associated with jets with $p_{\mathrm{T}}$ between 20 and $100 \mathrm{GeV}$ : (a) clusters per jet, (b) associated tracks with $p_{\mathrm{T}}>0.5 \mathrm{GeV}$, (c) associated tracks with $p_{\mathrm{T}}>1.0$ $\mathrm{GeV}$.

The jet shape variable $\rho(r)$ is defined by:

$$
\rho(r)=\left\langle\frac{1}{r} \frac{d p_{T}}{d r}\right\rangle_{j e t s}=\frac{1}{A} \frac{1}{N_{j e t}} \sum_{\text {jets }} p_{T}(r-\Delta r / 2, r+\Delta r / 2)
$$

i.e. the sum of constituent momenta in an annulus at distance $r$ from the jet axis, divided by the area of that annulus, and normalized to the number of jets. This variable is shown in figure 2 for jets with $p_{\mathrm{T}}>60 \mathrm{GeV}$, collected using the lowest-threshold jet trigger. More information on this comparison can be found in reference [9].

\section{Charged particle jet measurements}

The properties of charged particle jets are investigated for $p_{\mathrm{T}}>4 \mathrm{GeV}$ and $|\eta|<0.57$. Charged particle jets are formed by applying the jet algorithm to all primary charged particles with transverse momentum $p_{\mathrm{T}}>500 \mathrm{MeV}$. Primary charged particles are defined as charged particles 


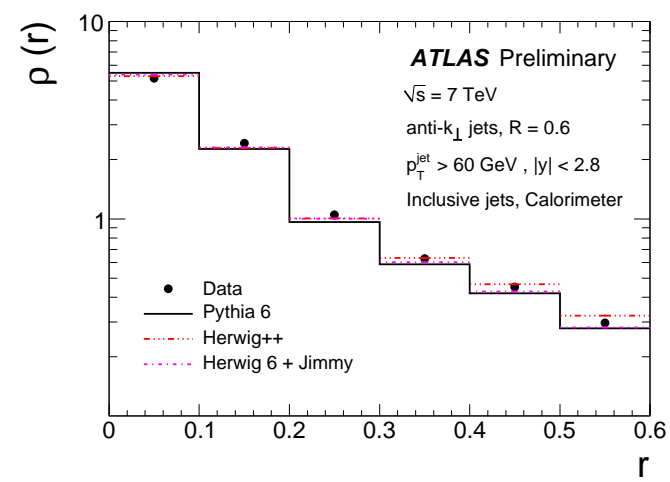

(a) Clusters

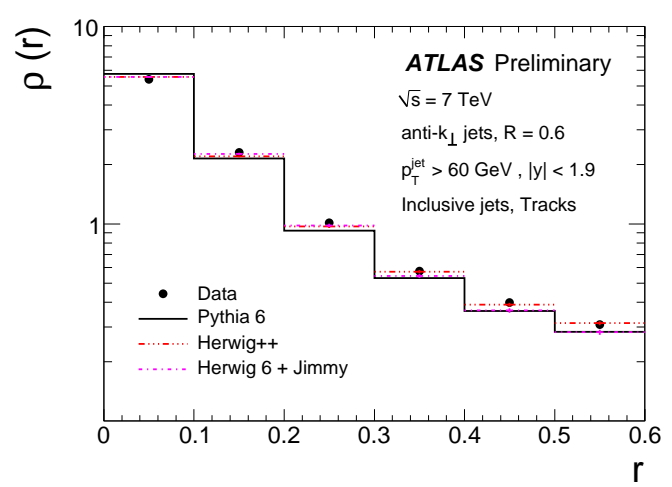

(b) Tracks

Figure 2: The uncorrected jet shape measured using (a) calorimeter clusters, or (b) tracks for anti- $k_{t}$ jets with $\mathrm{R}=0.6$, as a function of $\mathrm{r}$. All calorimeter jets with $p_{\mathrm{T}}>60 \mathrm{GeV}$ are included.

with a mean lifetime $\tau>0.3 \times 10^{-10}$ s produced in pp interactions or from subsequent decays of particles with a shorter lifetime.

This analysis uses data from an initial period of ATLAS running with a total luminosity of about $370 \mu \mathrm{b}^{-1}$. Events are selected with at least one MBTS hit and a reconstructed primary vertex. The efficiency of both the trigger and vertexing were determined from data to be negligibly different from $100 \%$. In these events, tracks were selected as described in section 3 and track jets reconstructed by applying the anti- $k_{t}$ algorithm as described in section 4 .

Two quantities are measured for these track jets: the inclusive cross section as a function of momentum, and the fragmentation for tracks with respect to each jet. The latter variable is defined by:

$$
z=\frac{\vec{p}_{\text {Track }} \cdot \hat{p}_{J e t}}{\left|\vec{p}_{J e t}\right|}
$$

Both the inclusive $p_{\mathrm{T}}$ and the $z$ cross-sections have detector effects unfolded. For the inclusive cross section, an unfolding procedure is used that corrects for jet-finding efficiency, reconstructed track jets not matched to a charged particle jet, and bin-to-bin migration of reconstructed jets due to tracking efficiency and resolution smearing. The ATLAS MC09 tune of Pythia 6 is used to derive the baseline corrections; charged particle jets are matched to reconstructed track jets by requiring $\Delta R<0.3$. Efficiencies and bin-to-bin migration are corrected via the Bayesian Iterative Unfolding algorithm [10]. Results are shown in figure 3 for $\mathrm{R}=0.4$ and 0.6 , with comparison to a range of Monte Carlo generators and tunes described in [11]. For the fragmentation measurement, $z$ distributions are produced in ranges of jet $p_{\mathrm{T}}$, and simple bin-by-bin correction factors are computed from Monte Carlo and applied to the data. Results are shown in figure 4 for $\mathrm{R}=0.6$ for selected jet momentum bins; plots for other 
bins and for $\mathrm{R}=0.4$ are available in reference [11].

Systematic uncertainties for tracking efficiency and fragmentation/underlying event effects apply to both the cross section and fragmentation measurements. These uncertainties are computed from an ensemble of toy Monte Carlo samples of the same size as the data are each unfolded using the same Pythia MC09 sample to produce the corrections. The average impact of a given uncertainty is determined by considering the deviation from the true answer, after unfolding, for each sample in an ensemble. The largest difference in unfolding performance observed for all Monte Carlo samples considered for this evaluation of the systematic error, is used as systematic uncertainty. Uncertainties due to tracking efficiency are evaluated by varying tracking efficiencies by $3 \%$. Uncertainties associated with Monte Carlo modeling, including tuning method, shower ordering, and varying parton distribution functions, are determined by using a range of Pythia 6 tunes.

For the cross section measurement, uncertainties associated with mismeasured high- $p_{\mathrm{T}}$ tracks, $\phi$ modelling, and an overall $11 \%$ luminosity uncertainty apply. More details on systematic error computation and validation can be found in in [11].

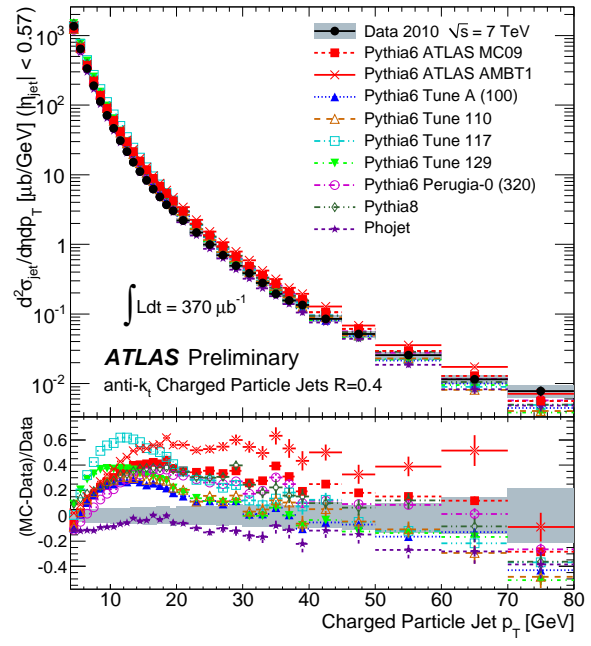

(a) $\mathrm{R}=0.4$

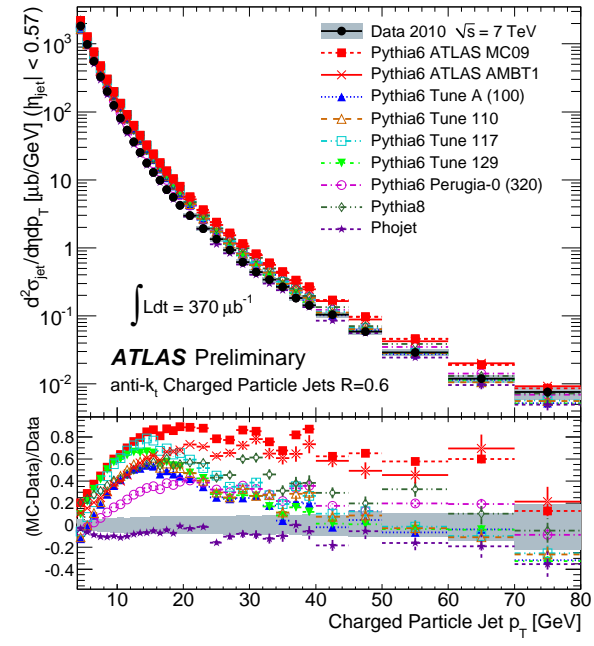

(b) $\mathrm{R}=0.6$

Figure 3: Corrected jet cross section for anti- $k_{t}$ jets with $\mathrm{R}=0.4$ and $\mathrm{R}=0.6$, compared with several Pythia 6 tunes, as well as Phojet and Pythia 8. The shaded area is the total uncertainty for the corrected data distribution, excluding the overall $11 \%$ luminosity uncertainty.

\section{Conclusions}

The first ATLAS studies of jet constituents have been shown. The number of raw constituents per reconstructed jet is in fair agreement with simulation, and improves for constituent $p_{\mathrm{T}}>1$ 


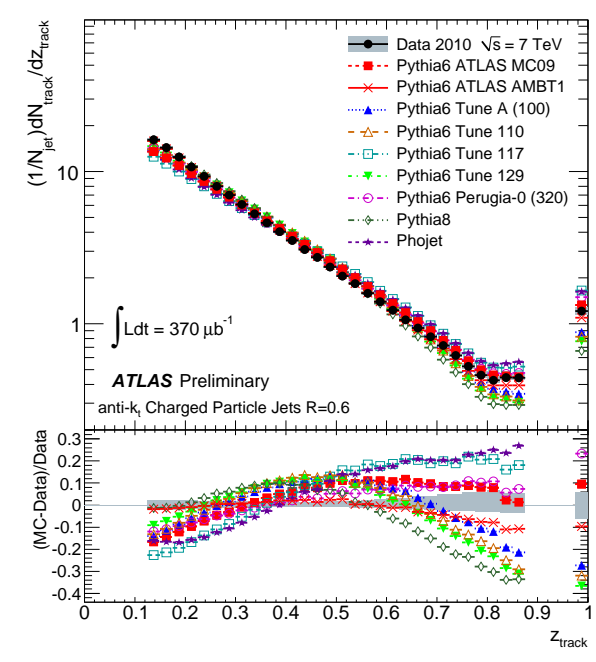

(a) $4 \mathrm{GeV}-6 \mathrm{GeV}$

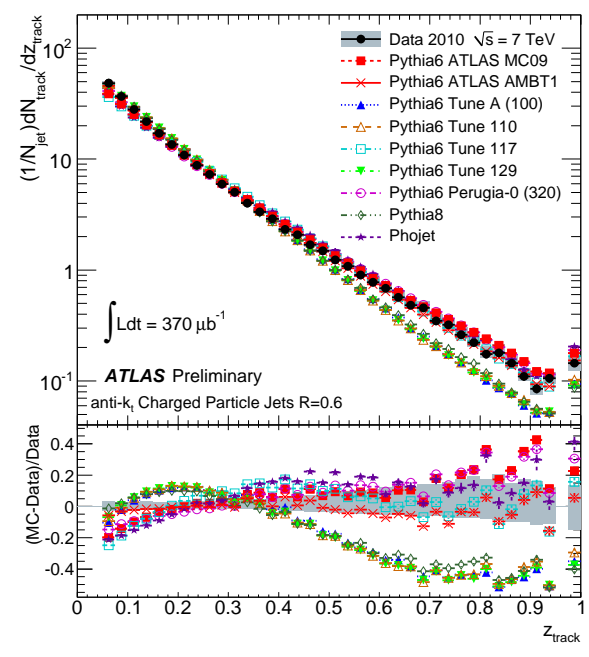

(b) $10 \mathrm{GeV}-15 \mathrm{GeV}$

Figure 4: Corrected fragmentation function in anti- $k_{t}$ jets with $\mathrm{R}=0.6$ in ranges of charged jet $p_{\mathrm{T}}$, compared with several Pythia 6 tunes, as well as Phojet and Pythia 8 . The shaded area is the total uncertainty for the corrected data distribution.

GeV. Jet shapes determined from calorimeter clusters and associated tracks likewise agree well with simulation.

The inclusive cross section of charged particle jets has been measured, and is best modeled by the Phojet event generator. The fragmentation for these jets is best modelled by the ATLAS AMBT1 [12] tune of Pythia 6. Both measurements provide data for further tuning of generators.

\section{References}

[1] ATLAS Collaboration, G. Aad et al., The ATLAS Experiment at the CERN Large Hadron Collider. JINST 3 (2008) S08003.

[2] T. Cornelissen et al., The new ATLAS track reconstruction (NEWT), J. Phys. Conf. Ser. 119 (2008) 032014.

[3] M. Cacciari, G. P. Salam, and G. Soyez, The anti-kt jet clustering algorithm, JHEP 04 (2008) 063.

[4] W. Lampl et al., Calorimeter Clustering algorithms: Description and Performance, Tech. Rep. ATLASLARG-PUB-2008-002, CERN, Geneva, December, 2008.

[5] ATLAS Collaboration, Properties of Jets and Inputs to Jet Reconstruction and Calibration with the ATLAS Detector Using Proton-Proton Collisions at $\sqrt{s}=7$ TeV, ATLAS-CONF-2010-053.

[6] A. Buckley, H. Hoeth, H. Lacker, H. Schulz, and J. E. von Suggern, Systematic event generator tuning for the LHC, Eur. Phys. J C65 (2010) 331.

[7] T. Sjorstrand, S. Mrenna, and P. Skands, PYTHIA6.4 Physics and Manual, JHEP 05 (2008) 026.

[8] S. Agostinelli et al., GEANT4: A simulation toolkit, Nucl. Instrum. Meth A506 (2003) 250. 
[9] ATLAS Collaboration, Measurement of jet production in proton-proton collisions at 7 Te $V$ centre-of-mass energy with the ATLAS detector, ATLAS-CONF-2010-050.

[10] G. D'Agostini, A Multidimensional unfolding method based on Bayes' theorem, Nucl. Instrum. Meth. A362 (1995) 487-498.

[11] ATLAS Collaboration, Measurement of differential cross section and fragmentation of jets from tracks in proton-proton collisions at center-of-mass energy $\sqrt{s}=7$ TeV with the ATLAS detector, ATLAS-CONF2010-049.

[12] ATLAS Collaboration, Charged particle multiplicities in pp interactions at sqrt(s) $=0.9$ and 7 TeV in a diffractive limited phase-space measured with the ATLAS detector at the LHC and new PYTHIA6 tune, ATLAS-CONF-2010-031. 